\title{
Magnetic-field-induced deflection of nonlocal light bullets in a Rydberg atomic gas
}

\author{
Xiujia Dong (董秀佳) ${ }^{1}$, Yao Ding (丁 瑶) $)^{1}$, Zhengyang Bai (白正阳 $)^{*^{*}}$, and Guoxiang Huang (黄国翔) $)^{1,2^{* *}}$ \\ ${ }^{1}$ State Key Laboratory of Precision Spectroscopy, Shanghai 200062, China \\ ${ }^{2}$ NYU-ECNU Institute of Physics at NYU Shanghai, Shanghai 200062, China \\ *Corresponding author: zhybai@|ps.ecnu.edu.cn \\ ${ }^{*}$ Corresponding author: gxhuang@phy.ecnu.edu.cn \\ Received December 15, 2021 | Accepted January 25, 2022 | Posted Online February 28, 2022
}

\begin{abstract}
Light bullets (LBs) are localized nonlinear waves propagating in high spatial dimensions. Finding stable LBs and realizing their control are desirable due to the interesting physics and potential applications. Here, we show that nonlocal LBs generated in a cold Rydberg atomic gas via the balance among the dispersion, diffraction, and giant nonlocal Kerr nonlinearity contributed by long-range Rydberg-Rydberg interaction can be actively manipulated by using a weak gradient magnetic field. Nonlocal LBs are generated by a balance among dispersion, diffraction, and large nonlocal Kerr nonlinearities contributed by long-range Rydberg-Rydberg interactions. Here, we find that active manipulation can be achieved by weak gradient magnetic fields in cold Rydberg atomic gases. Especially, the LBs may undergo significant Stern-Gerlach deflections, and their motion trajectories can be controlled by adjusting the magnetic-field gradient. The results reported here are helpful not only for understanding unique properties of LBs in nonlocal optical media but also for finding ways for precision measurements of magnetic fields.
\end{abstract}

Keywords: nonlinear optics; optical soliton; Rydberg atom. DOI: 10.3788/COL202220.041902

\section{Introduction}

In recent years, much attention has been paid to the investigation on electromagnetically induced transparency (EIT) in cold Rydberg atomic gases ${ }^{[1-26]}$. This is rooted in the fact that atomic Rydberg states have long coherent lifetimes and strong longrange interaction (called Rydberg-Rydberg interaction) between remote atoms. The Rydberg-Rydberg interaction makes the atomic gases be nonlocal optical media, and they can be effectively mapped to strong photon-photon interactions via EIT. As a result, strong nonlinearities at very low light intensity can be realized ${ }^{[5,6,14]}$, which opens up a new avenue to study nonlinear and quantum optics and realize novel photon devices, such as single-photon switches ${ }^{[27-31]}$, optical transistors ${ }^{[4,32]}$, photon memories ${ }^{[10,11]}$, and single-photon sources ${ }^{[33]}$.

Light bullets $(\mathrm{LBs})^{[34]}$ are solitary nonlinear waves localized in $m$ spatial dimensions and one time dimension $[(m+1) \mathrm{D}$; $m=1,2,3]$. In recent years, the study of LBs has attracted intensive theoretical and experimental interests ${ }^{[35]}$ because of their rich nonlinear physics and technological applications ${ }^{[36,37]}$. However, the generation of stable high-dimensional LBs is a topic not solved for a long time. It has been shown recently that stable $(3+1) \mathrm{D}$ nonlocal LBs can be realized in Rydberg atomic gases; such LBs have extremely low generation power and ultra slow propagation velocity ${ }^{[17]}$. Different from the non-interaction system, the central element is the co-existence of giant nonlocal and local optical Kerr nonlinearities. The former features a fast (sub-microsecond) response ${ }^{[38]}$, which is complemented by the latter, whose response is relatively slow (in the order of microseconds). In conjunction with tunable dispersion and diffraction, this allows us to precisely control dynamics of LBs.

In this work, we propose a scheme to realize the active control of the nonlocal LBs in a Rydberg atomic gas. We show that the $(3+1) \mathrm{D}$ LBs generated in such a system via EIT can be manipulated by using a gradient magnetic field. In particular, the LBs can undergo significant Stern-Gerlach deflections even when the magnetic-field gradient is weak, and their motion trajectories can be adjusted through the changing of the magnetic-field gradient. Our work contributes to the efforts for understanding the unique properties and realizing the active controls of highdimensional LBs and also for finding new techniques for precision measurements of magnetic fields.

\section{Model}

The system under study is a cold three-level atomic gas working with a Rydberg-EIT scheme, shown in Fig. 1(a). Here, the levels 
(a)

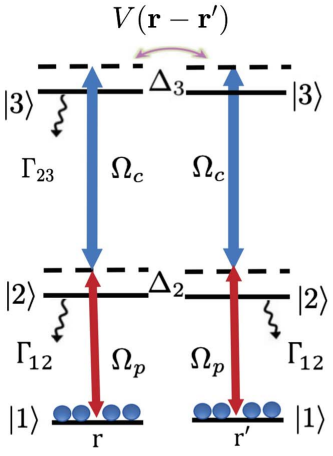

(b)

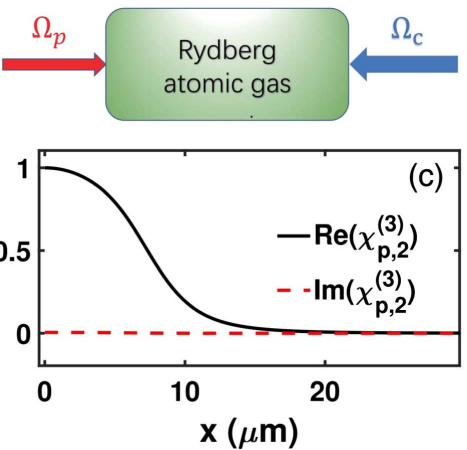

$\mathrm{z} /\left(2 \mathrm{~L}_{\text {diff }}\right)=$

(d1) LB
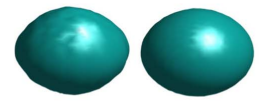

(d2) Vortex

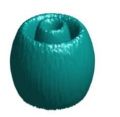

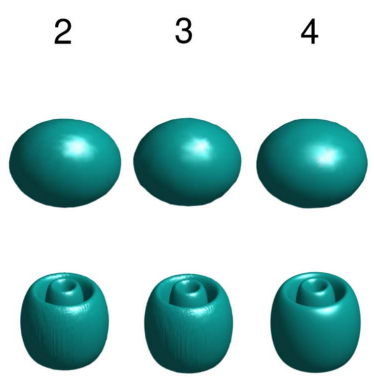

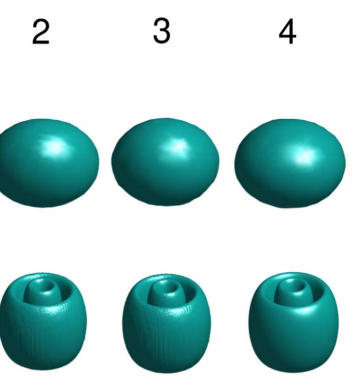

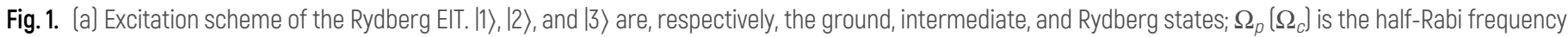
of the probe (control) laser field; $\Gamma_{12}(\sim \mathrm{MHz})$ and $\Gamma_{23}(\sim \mathrm{kHz})$ are, respectively, decay rates from $|2\rangle$ to $|1\rangle$ and $|3\rangle$ to $|2\rangle_{;} \Delta_{2}=\omega_{p}-\left(\omega_{2}-\omega_{1}\right)$ and $\Delta_{3}=\omega_{p}+\omega_{C}-$ $\left(\omega_{c}-\omega_{1}\right)$ are, respectively, the one- and two-photon detunings. $\hbar\left(r r-r^{\prime}\right)$ is the vdW interaction between the two atoms in Rydberg states, respectively, located at $r$ and $r^{\prime}$. (b) Geometry of the system. The probe and control fields counter-propagate in the Rydberg atomic gas. (c) Normalized $\chi_{p, 2}^{(3)}$ (i.e., the coefficient of nonlocal Kerr nonlinearity) as a function of coordinate $x$, with the solid black (dashed red) line representing its real part Re $\left(\chi_{p, 2}^{(3)}\right.$ ) [imaginary part $\left.\operatorname{Im}\left(\chi_{p, 2}^{(3)}\right)\right]$ for coordinate $y=0$ (see text for more details). Evolution of a (d1) nonlocal LB and (d2) vortex in the system.

$|1\rangle,|2\rangle$, and $|3\rangle$ are ground, intermediate, and Rydberg states, respectively; a weak, pulsed (with time duration $\tau_{0}$ ) probe laser field (center angular frequency $\omega_{p}$, center wavenumber $k_{p}=\omega_{p} / c$, half-Rabi frequency $\Omega_{p}$ ) couples to the transition between $|1\rangle$ and $|2\rangle$; a strong, continuous-wave control laser field (angular frequency $\omega_{c}$, wavenumber $k_{c}=\omega_{c} / c$, half-Rabi frequency $\Omega_{c}$ ) couples to the transition between $|2\rangle$ and $|3\rangle$. The total electric field of the system reads $\mathbf{E}=\mathbf{E}_{p}+\mathbf{E}_{c}$, with $\mathbf{E}_{\alpha}=\mathbf{e}_{\alpha} \mathcal{E}_{\alpha} \exp \left[i\left(\mathbf{k}_{\alpha} \cdot \mathbf{r}-\omega_{\alpha} t\right)\right]+$ h.c.. Here, with $\mathbf{r}=(x, y, z), \mathbf{e}_{\alpha}$ and $\mathcal{E}_{\alpha}$ are the unit polarization vector and field amplitude for the $\alpha$ field $(\alpha=p, c)$, respectively. To suppress Doppler effect, the probe and the control fields are assumed to counter-propagate along the $z$ direction. Figure 1(b) shows the geometry of the system.

For realizing the active control on the LBs, a weak gradient magnetic field is assumed to act on the atomic gas, with the form

$$
\mathbf{B}(x, y)=\hat{\mathbf{z}} B\left(\mathbf{r}_{\perp}\right)=\hat{\mathbf{z}}\left(B_{1} x+B_{2} y\right),
$$

where $\mathbf{r}_{\perp}=(x, y, 0), \hat{\mathbf{z}}=(0,0,1)$ is the unit vector in the $z$ direction, $B_{1}$ and $B_{2}$ characterize the gradients of the magnetic field in the $x-y$ plane. Due to the presence of the magnetic field, each level of the atoms is split into a series of Zeeman sub-levels with energy $\Delta E_{\alpha}^{Z \text { Zeeman }}=\mu_{B} g_{F}^{\alpha} m_{F}^{\alpha} B$, where $\mu_{B}, g_{F}^{\alpha}$, and $m_{F}^{\alpha}$ are the Bohr magneton, gyromagnetic factor, and magnetic quantum number of level $|\alpha\rangle$, respectively. As a result, the one- and two-photon detunings $\Delta_{2}$ and $\Delta_{3}$ are changed into $\Delta_{2}\left(\mathbf{r}_{\perp}\right)=\left(\omega_{p}-\omega_{c}-\right.$ $\left.\omega_{21}\right)+\mu_{21} B\left(\mathbf{r}_{\perp}\right)$ and $\Delta_{3}\left(\mathbf{r}_{\perp}\right)=\left(\omega_{p}-\omega_{31}\right)+\mu_{31} B\left(\mathbf{r}_{\perp}\right)$, with $\mu_{\alpha \beta}=\mu_{B}\left(g_{F}^{\alpha} m_{F}^{\alpha}-g_{F}^{\beta} m_{F}^{\beta}\right) / \hbar$.

Under electric-dipole and rotating-wave approximations, the Hamiltonian of the atomic gas including the Rydberg-Rydberg interaction is given by $\hat{H}=\mathcal{N}_{a} \int \mathrm{d}^{3} r \hat{\mathcal{H}}(\mathbf{r}, t)$, with $\hat{\mathcal{H}}(\mathbf{r}, t)$ the Hamiltonian density, given by

$$
\begin{aligned}
\hat{\mathcal{H}}= & \sum_{\alpha=1}^{3} \hbar \omega_{\alpha} \hat{S}_{\alpha \alpha}(\mathbf{r}, t)-\hbar\left[\Omega_{p} \hat{S}_{12}(\mathbf{r}, t)+\Omega_{c} \hat{S}_{23}(\mathbf{r}, t)+\text { h.c. }\right] \\
& +\mathcal{N}_{a} \int \mathrm{d}^{3} \mathbf{r}^{\prime} \hat{S}_{33}\left(\mathbf{r}^{\prime}, t\right) \hbar V\left(\mathbf{r}^{\prime}-\mathbf{r}\right) \hat{S}_{33}(\mathbf{r}, t) .
\end{aligned}
$$

Here, $\mathcal{N}_{a}$ is atomic density, $\Omega_{p} \equiv\left(\mathbf{e}_{p} \cdot \mathbf{p}_{21}\right) \mathcal{E}_{p} / \hbar$ and $\Omega_{c} \equiv$ $\left(\mathbf{e}_{c} \cdot \mathbf{p}_{32}\right) \mathcal{E}_{c} / \hbar$ are, respectively, the half-Rabi frequencies of the probe and control fields (with $\mathbf{p}_{\alpha \beta}$ the electric-dipole matrix element associated with the transition from $|\beta\rangle$ to $|\alpha\rangle), \hat{S}_{\alpha \beta} \equiv$ $|\beta\rangle\langle\alpha| e^{i\left[\left(\mathbf{k}_{\beta}-\mathbf{k}_{\alpha}\right) \cdot \mathbf{r}-\left(\omega_{\beta}-\omega_{\alpha}+\Delta_{\beta}-\Delta_{\alpha}\right) t\right]}$ are atomic transition operators $(\alpha, \beta=1,2,3)$, and $\hbar V\left(\mathbf{r}-\mathbf{r}^{\prime}\right) \equiv-\hbar C_{6} /\left|\mathbf{r}-\mathbf{r}^{\prime}\right|^{6}$ is the van der Waals (vdW) interaction potential (with $C_{6}$ the dispersion coefficient) between the Rydberg atoms located, respectively, at the positions $\mathbf{r}$ and $\mathbf{r}^{\prime[17]}$.

The dynamics of the atoms is controlled by the Heisenberg equation of motion for the atomic operators $\hat{S}_{\alpha \beta}$, i.e., $i \hbar \partial \hat{S}_{\alpha \beta} / \partial t=$ $\left[\hat{H}, \hat{S}_{\alpha \beta}\right]$. Taking expectation values on both sides of this equation, we obtain the optical Bloch equation involving one- and twobody reduced density matrices (DMs), which can be cast into the form

$$
\frac{\partial \rho}{\partial t}=-\frac{i}{\hbar}[\hat{H}, \rho]-\Gamma[\rho]
$$

Here, $\rho(\mathbf{r}, t)$ is the reduced DM in the single-particle basis $\{|1\rangle, 2\rangle, 3\rangle\}$, with the matrix elements defined by $\rho_{\alpha \beta}(\mathbf{r}, t) \equiv\left\langle\hat{S}_{\alpha \beta}(\mathbf{r}, t)\right\rangle ; \Gamma$ is a $3 \times 3$ relaxation matrix describing the spontaneous emission and dephasing. Due to the existence of the Rydberg-Rydberg interaction, two-body reduced DM [i.e., $\rho_{\text {twobody }}$ with DM elements $\left.\rho_{\alpha \beta, \mu \nu}\left(\mathbf{r}^{\prime}, \mathbf{r}, t\right)\right]$ is involved in this equation, denoting the contribution from the Rydberg-Rydberg interaction. The explicit expression of Eq. (3) is presented in Supplementary Material ${ }^{[39]}$. 
The dynamics of the probe field is described by the Maxwell equation, which, under the slowly varying envelope approximation, reads

$$
i\left(\frac{\partial}{\partial z}+\frac{1}{c} \frac{\partial}{\partial t}\right) \Omega_{p}+\frac{c}{2 \omega_{p}} \nabla_{\perp}^{2} \Omega_{p}+\kappa_{12} \rho_{21}=0,
$$

where $\nabla_{\perp}^{2}=\partial^{2} / \partial x^{2}+\partial^{2} / \partial y^{2}, \kappa_{12}=\mathcal{N}_{a} \omega_{p}\left|\mathbf{p}_{12}\right|^{2} /\left(2 \varepsilon_{0} c \hbar\right)$, with $c$ as the light speed in vacuum. For a relatively weak probe field, the population in atomic levels changes not much when the probe field is applied to the system, and hence a perturbation expansion beyond mean-field approximation for many-atom correlations can be employed to solve the Bloch equation, Eq. $(3)^{[6,14,17]}$. The expression of the nonlinear optical susceptibility of the exact probe field to the third order of the perturbation expansion is given by

$$
\chi_{p} \simeq \chi_{p}^{(1)}+\chi_{p, 1}^{(3)}\left|\mathcal{E}_{p}\right|^{2}+\int \mathrm{d} \mathbf{r}_{\perp}^{\prime} \chi_{p, 2}^{(3)}\left(\mathbf{r}_{\perp}-\mathbf{r}_{\perp}^{\prime}\right)\left|\mathcal{E}_{p}\left(\mathbf{r}_{\perp}^{\prime}\right)\right|^{2},
$$

where $\chi_{p}^{(1)}=\mathcal{N}_{a}\left|\mathbf{p}_{12}\right|^{2} a_{21}^{(1)} /\left(\varepsilon_{0} \hbar\right), \quad \chi_{p, 1}^{(3)}=\mathcal{N}_{a}\left|\mathbf{p}_{12}\right|^{4} a_{21,1}^{(3)} /\left(\varepsilon_{0} \hbar^{3}\right)$, and $\chi_{p, 2}^{(3)}=\int \mathrm{d} z \mathcal{N}_{a}^{2}\left|\mathbf{p}_{12}\right|^{4} a_{21,2}^{(3)} /\left(\varepsilon_{0} \hbar^{3}\right)^{[14,17]}$. In the above expression, the third term is the nonlocal Kerr nonlinear susceptibility, contributed from the Rydberg-Rydberg interaction, while the second term is the local Kerr nonlinear susceptibility, contributed from the non-zero two-photon detuning (i.e., $\Delta_{3} \neq 0$ ).

To be concrete in the following calculations, we choose strontium atoms $\left({ }^{88} \mathrm{Sr}\right)$, although our approach is valid for other Rydberg atomic gases. The energy levels shown in Fig. 1(a) are selected as $|1\rangle=\left|5 \mathrm{~s}^{21} \mathrm{~S}_{0}\right\rangle,|2\rangle=\left|5 \mathrm{~s} 5 \mathrm{p}^{1} \mathrm{P}_{1}\right\rangle,|3\rangle=\left|5 \mathrm{~s} n \mathrm{~s}^{1} \mathrm{~S}_{0}\right\rangle$, with $n$ the principal quantum number ${ }^{[39]}$. The spontaneous emission rates of the atoms are given by $\Gamma_{12}=2 \pi \times 16 \mathrm{MHz}$, $\Gamma_{23}=2 \pi \times 16.7 \mathrm{kHz}$, so one has $\gamma_{21}=\Gamma_{12} / 2, \gamma_{31}=\Gamma_{23} / 2$, and $\gamma_{32}=\left(\Gamma_{12}+\Gamma_{23}\right) / 2$. For this choice, the vdW interaction in ${ }^{1} \mathrm{~S}_{0}$ states is isotropically attractive $\left(C_{6}>0\right)$, which is important to realize self-focusing nonlocal Kerr nonlinearity.

The result shown in Fig. 1(c) is $\chi_{p, 2}^{(3)}$ as a function of $x$ for $y=0$. The real part $\left[\operatorname{Re}\left(\chi_{p, 2}^{(3)}\right)\right]$ and imaginary part $\left[\operatorname{Im}\left(\chi_{p, 2}^{(3)}\right)\right]$ are plotted on the condition that $\Delta_{2} \gg \Gamma_{12}$ (i.e., the system works in the dispersive nonlinearity regime) by the solid black line and the dashed red line, respectively. We see that $\operatorname{Im}\left(\chi_{p, 2}^{(3)}\right)$ is much smaller than $\operatorname{Re}\left(\chi_{p, 2}^{(3)}\right)$, which means that the optical absorption of the probe field is negligible, resulting from the EIT effect and the condition of large one-photon detuning; moreover, $\operatorname{Re}\left(\chi_{p, 2}^{(3)}\right)$ is an attractive potential well, and there is a saturation near $x=0$, which is due to the Rydberg blockade effect (with blockade radius $\sim 7 \mu \mathrm{m}$ ) that suppresses the excitation of atoms to the Rydberg state and hence causes the nonlinear kernel $\chi_{p, 2}^{(3)}$ to saturate to a finite value. By virtue of the strong Rydberg-Rydberg interaction, the nonlocal optical nonlinearities can reach $\int \mathrm{d} \mathbf{r}_{\perp}^{\prime} \chi_{p, 2}^{(3)}\left(\mathbf{r}_{\perp}^{\prime}\right) \sim 10^{-8} \mathrm{~m}^{2} \mathrm{~V}^{-2}$, which are many orders of magnitude larger than that of conventional EIT systems ${ }^{[6,14]}$.

\section{3. $(3+1)$ D Nonlinear Envelope Equation}

Our main aim is to implement an active control of LBs in the system. To make the related physical mechanism transparent, we first derive the equation describing the nonlinear evolution of the probe-field envelope. For a modulated plane-wave of the probe field, we assume $\Omega_{p} \sim F \exp [i(K z-\omega t)]^{[40]}$. The equation for the envelope $F$ in the presence of the magnetic field can be derived by means of the multiple-scale perturbation method, similar to that carried out in Ref. [41]. We obtain

$$
\begin{aligned}
i \frac{\partial u}{\partial s}= & -\left(\frac{\partial^{2}}{\partial \xi^{2}}+\frac{\partial^{2}}{\partial \eta^{2}}\right) u-g_{d} \frac{\partial^{2} u}{\partial \sigma^{2}}-V_{m}(\xi, \eta) u \\
& +\left[W_{1}|u|^{2}+\int \mathrm{d} \tilde{\mathbf{r}}_{\perp} W_{2}\left(\tilde{\mathbf{r}}_{\perp}-\tilde{\mathbf{r}}_{\perp}^{\prime}\right)\left|u\left(\tilde{\mathbf{r}}_{\perp}^{\prime}, s\right)\right|^{2}\right] u+i d_{0} u
\end{aligned}
$$

Here, $s=z /\left(2 L_{\mathrm{diff}}\right) ; \tilde{\mathbf{r}}_{\perp}=(\xi, \eta)=(x, y) / R_{0} ; \sigma \equiv\left(t-z / V_{g}\right) / \tau_{0}$ $\left[V_{g} \equiv(\partial K / \partial \omega)^{-1}\right.$ is group velocity, with $K \equiv K(\omega)$ the linear dispersion relation]; $u=\left(F / U_{0}\right) \exp \left(-\alpha_{0} z\right)$, with $\alpha_{0} \equiv \operatorname{Im}(K)$ a decay constant; $g_{d} \equiv-L_{\text {diff }} K_{2} / \tau_{0}^{2}, W_{1} \equiv-b k_{p}^{2} R_{0}^{2} U_{0}^{2} \chi_{p, 1}^{(3)}, W_{2} \equiv$ $-b k_{p}^{2} R_{0}^{4} U_{0}^{2} \chi_{p, 2}^{(3)}$, and $d_{0} \equiv-2 L_{\text {diff }} / L_{A}$ are dimensionless coefficients of dispersion, local Kerr nonlinearity, nonlocal Kerr nonlinearity, and absorption, respectively. In these coefficients, $b=\hbar /\left|\mathbf{p}_{12}\right|^{2}, K_{2} \equiv \partial^{2} K / \partial \omega^{2}$ describes group-velocity dispersion, $L_{\text {diff }} \equiv k_{p} R_{0}^{2}$ and $L_{A} \equiv 1 / \alpha_{0}$ are, respectively, the typical diffraction and absorption lengths, and $U_{0}$ and $R_{0}$ are, respectively, the typical half-Rabi frequency and transverse size of the probe field. Since we are interested only in the dispersive nonlinearity regime of the system, where the $L_{A}$ is much larger than the other typical lengths, and hence $d_{0}$ is very small, the imaginary parts of the coefficients in Eq. (6) are negligible.

In Eq. (6), $V_{m} \equiv-k_{p}^{2} R_{0}^{2} \chi_{p}^{(1)}$ is a dimensionless linear potential contributed by the gradient magnetic field. It has the form $V_{m}(\xi, \eta)=V_{1} \xi+V_{2} \eta$, with

$$
\begin{gathered}
V_{1}=\frac{\kappa_{12} R_{0} L_{\mathrm{diff}}\left[\left(\omega+\Delta_{3}\right) \Delta_{3} \mu_{21}+\left|\Omega_{c}\right|^{2} \mu_{31}\right]}{\left[\left(\omega+\Delta_{2}\right)\left(\omega+\Delta_{3}\right)-\left|\Omega_{c}\right|^{2}\right]^{2}} B_{1}, \\
V_{2}=\frac{\kappa_{12} R_{0} L_{\mathrm{diff}}\left[\left(\omega+\Delta_{3}\right) \Delta_{3} \mu_{21}+\left|\Omega_{c}\right|^{2} \mu_{31}\right]}{\left[\left(\omega+\Delta_{2}\right)\left(\omega+\Delta_{3}\right)-\left|\Omega_{c}\right|^{2}\right]^{2}} B_{2} .
\end{gathered}
$$

We then consider the formation of LBs when the gradient magnetic field is absent (i.e., $\left.V_{m}=0\right)$. In this case, stable ( $3+$ 1)D LBs and vortices can form, with the result by a numerical simulation shown in Fig. 1(d). From the figure, we see that the $(3+1)$ D LB (upper part) and vortex (lower part) relax to self-cleaned forms quite close to the unperturbed ones ${ }^{[42]}$, and their shapes undergo no apparent change during propagation. The physical parameters used in the simulation are chosen as $\Delta_{2}=-15 \Gamma_{12}, \Delta_{3}=-0.02 \Gamma_{12}, R_{0}=10 \mu \mathrm{m}, \tau_{0}=9 \times 10^{-7} \mathrm{~s}, \mathcal{N}_{a}=$ $3 \times 10^{10} \mathrm{~cm}^{-3}, U_{0}=0.3 \Gamma_{12}$, and $C_{6} \simeq 2 \pi \times 81.6 \mathrm{MHz} \mu \mathrm{m}^{6}$ (for the principal quantum number $n=60$ ). With these parameters, we obtain $L_{\text {diff }}=1.36 \mathrm{~mm}, L_{A}=907 \mathrm{~mm}, R_{b}=6 \mu \mathrm{m}, g_{d}=0.134$, and $d_{0}=-0.03$. Such an LB can form in a very short distance 
and generate extremely low light power $(\sim 1 \mathrm{nW})$, which is due to the giant Kerr nonlinearity (contributed by the RydbergRydberg interaction) and the ultraslow propagation velocity of the probe pulse $\left(\sim 2.3 \times 10^{-6} c\right.$, contributed from the EIT effect).

\section{Manipulation of LBS}

We now turn to investigate what will happen for a nonlocal LB when an external gradient magnetic field is present. As a first step, we consider Eq. (6) in the absence of the Kerr nonlinearity (i.e., $W_{1}=W_{2}=0$ ). Using the transformation $u=$ $u^{\prime} \exp \left[i\left(V_{1} \xi^{\prime}+V_{2} \eta^{\prime}+V_{1}^{2} s^{2} / 3+V_{2}^{2} s^{2} / 3\right) s\right]$, with $\quad \xi^{\prime}=\xi-$ $V_{1} s^{2} / 2$ and $\eta^{\prime}=\eta-V_{2} s^{2} / 2$, Eq. (6) is converted into the form $i \partial u^{\prime} / \partial s=-(1 / 2)\left(\partial^{2} / \partial \xi^{\prime 2}+\partial^{2} / \partial \eta^{\prime 2}+g_{d} \partial^{2} / \partial \tau^{2}\right) u^{\prime}$. It is easy to obtain the expression of the central position of the probe pulse in the $\xi-\eta$ plane, which is given by $(\xi, \eta)=\left(V_{1} s^{2} / 2, V_{2} s^{2} / 2\right)$. Returning to the original variables, the central position of the pulse reads

$$
(x, y)=\kappa_{12} \frac{\left[\left(\omega+\Delta_{3}\right) \Delta_{3} \mu_{21}+\left|\Omega_{c}\right|^{2} \mu_{31}\right]}{\left[\left(\omega+\Delta_{2}\right)\left(\omega+\Delta_{3}\right)-\left|\Omega_{c}\right|^{2}\right]^{2}} \frac{R_{0}^{2}}{L_{\mathrm{diff}}} z^{2}\left(B_{1}, B_{2}\right) .
$$

We see that, due to the presence of the magnetic field, the motion of the linear wave is changed, and its trajectory in the $x-$ $y$ plane has a deflection with a quadratic dependence on the propagation coordinate $z$; moreover, the trajectory can be controlled by tuning the gradient of the magnetic field, i.e., by manipulating the parameters $B_{1}$ and $B_{2}$.

In the presence of the Kerr nonlinearities, it is hard to get an exact expression for the motion trajectory of the probe pulse. In this situation, however, one can obtain the trajectory deflection by resorting to a numerical simulation for solving Eq. (6). Figure 2(a) shows the result of the 3D motion trajectory of an $\mathrm{LB}$ as a function of $x / R_{0}, y / R_{0}$, and $z /\left(2 L_{\text {diff }}\right)$, in the presence of the gradient magnetic field with $\left(B_{1}, B_{2}\right)=(3.2,0) \mathrm{mG} \mathrm{cm}^{-1}$. The corresponding trajectory in the $x-z$ plane is illustrated in Fig. 2(b). We see clearly that the LB experiences a deflection due to the existence of the magnetic field. Shown in Fig. 2(c) is the result of the 3D motion trajectory of the LB for an increased magnetic-field gradient in the $x$ direction by taking $\left(B_{1}, B_{2}\right)=(6.4,0) \mathrm{mG} \mathrm{cm}^{-1}$. One sees that the trajectory of the LB is changed significantly due to the increase of the magnetic field.

In addition, richer motion trajectories of the LB can be obtained by using different magnetic fields. To prove this, we consider a time-varying gradient magnetic field of the form

$$
\mathbf{B}(x, t)=\hat{\mathbf{z}} B(x, t)=\hat{\mathbf{z}} B_{0} \cos \left(\omega_{0} t\right) x,
$$

where $\omega_{0}$ characterizes the motion period of the magnetic field in time. Figure 3(a) shows the motion trajectory of the LB under the action of such a magnetic field. We see that the trajectory of the LB follows the variation of the magnetic field. Illustrated in Fig. 3(b) is the corresponding sinusoidal trajectory of the LB in
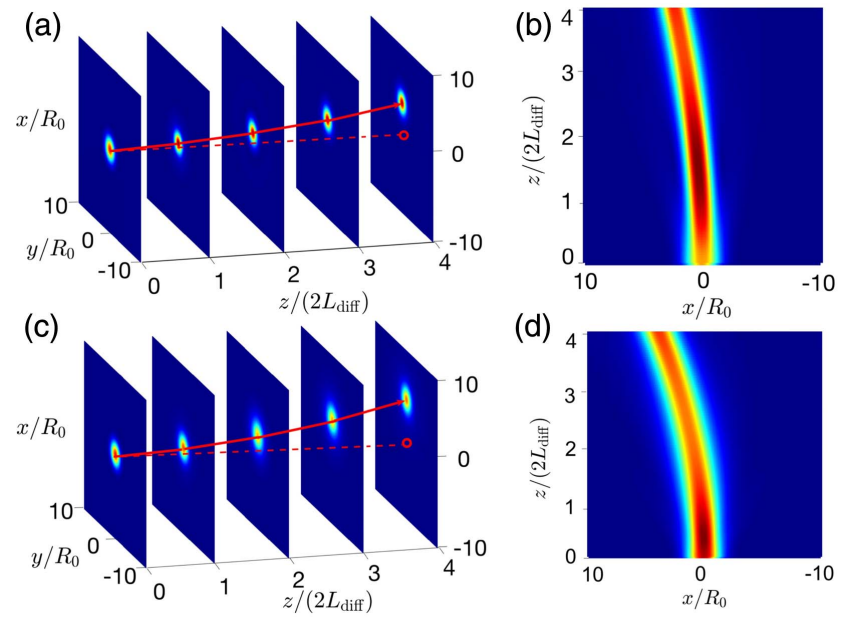

Fig. 2. Stern-Gerlach deflections of nonlocal LBs. (a) 3D motion trajectory of an $L B$ as a function of $x / R_{0}, y / R_{0}$, and $z /\left(2 L_{\text {diff }}\right)$ in the presence of the gradient magnetic field $\left(B_{1}, B_{2}\right)=(3.2,0) \mathrm{mG} \mathrm{cm}{ }^{-1}$; (C) 3D motion trajectory of the $L B$ for $\left(B_{1}, B_{2}\right)=(6.4,0) \mathrm{mG} \mathrm{cm}^{-1}$. (b) and (d) are trajectories of the $L B$ in the $x-z$ plane, corresponding, respectively, to panels (a) and (c).
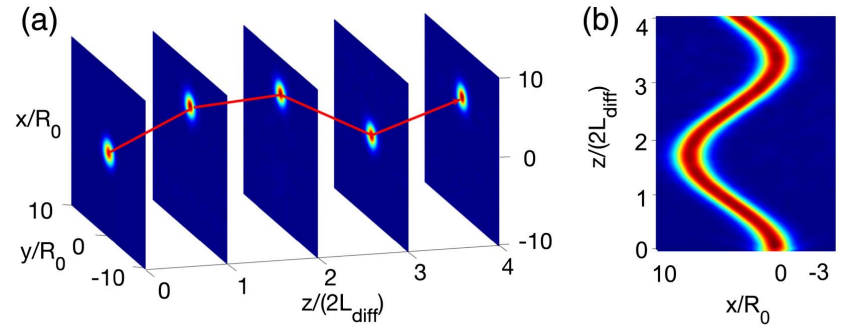

Fig. 3. Motion trajectory of the LB in the presence of a time-varying gradient magnetic field. (a) Trajectory of the LB as a function of $x / R_{0}, y /$ $R_{0}$, and $z /\left(2 L_{\text {diff }}\right)$ when the time-varying gradient magnetic field of Eq. (9) is present. (b) The corresponding sinusoidal trajectory of the LB in the $x-z$ plane.

the $x-z$ plane. Obviously, one can use various magnetic fields to manipulate the motion of LBs; conversely, the trajectory deflections of the LBs may be exploited to measure external magnetic fields.

\section{Conclusion}

We have shown that nonlocal LBs created in a cold Rydberg atomic gas can be actively manipulated by using a weak gradient magnetic field. In particular, the LBs can experience significant Stern-Gerlach deflections when a weak external magnetic field is applied, and their motion paths may be controlled through the adjustment of the magnetic-field gradient. The results reported here are useful not only for understanding novel properties of 
the LBs in nonlocal optical media but also for finding new ways for precision measurements of magnetic fields.

\section{Acknowledgement}

This work was supported by the National Natural Science Foundation of China (NSFC) (Nos. 11975098, 11174080, and 11904104) and the Shanghai Pujiang Program (No. 21PJ1402500).

\section{References and Notes}

1. M. Saffman, T. G. Walker, and K. Mфlmer, "Quantum information with Rydberg atoms," Rev. Mod. Phys. 82, 2313 (2010).

2. J. D. Pritchard, K. J. Weatherill, and C. S. Adams, "Nonlinear optics using cold Rydberg atoms," in Annual Review of Cold Atoms and Molecules, Vol. 1 (World Scientific, 2012), p. 301.

3. O. Firstenberg, C. S. Adams, and S. Hofferberth, "Nonlinear quantum optics mediated by Rydberg interactions," J. Phys. B 49, 152003 (2016).

4. C. Murray and T. Pohl, "Quantum and nonlinear optics in strongly interacting atomic ensembles," in Advances in Atomic, Molecular, and Optical Physics, Vol. 65, Chap. 7 (Academic, 2016), p. 321.

5. J. D. Pritchard, D. Maxwell, A. Gauguet, K. J. Weatherill, M. P. A. Jones, and C. S. Adams, "Cooperative atom-light interaction in a blockaded Rydberg ensemble," Phys. Rev. Lett. 105, 193603 (2010).

6. S. Sevinçli, N. Henkel, C. Ates, and T. Pohl, "Nonlocal nonlinear optics in cold Rydberg gases," Phys. Rev. Lett. 107, 153001 (2011).

7. D. Yan, C. Cui, Y. Liu, L. Song, and J. Wu, "Normal and abnormal nonlinear electromagnetically induced transparency due to dipole blockade of Rydberg excitation," Phys. Rev. A 87, 023827 (2013).

8. A. V. Gorshkov, J. Otterbach, M. Fleischhauer, T. Pohl, and M. D. Lukin, "Photon-photon interactions via Rydberg blockade," Phys. Rev. Lett. 107, 133602 (2011).

9. O. Firstenberg, T. Peyronel, Q. Liang, A. V. Gorshkov, M. D. Lukin, and V. Vuletić, "Attractive photons in a quantum nonlinear medium," Nature 502, 71 (2013).

10. D. Maxwell, D. J. Szwer, D. Paredes-Barato, H. Busche, J. D. Pritchard, A. Gauguet, K. J. Weatherill, M. P. A. Jones, and C. S. Adams, "Storage and control of optical photons using Rydberg polaritons," Phys. Rev. Lett. 110, 103001 (2013)

11. L. Li and A. Kuzmich, "Quantum memory with strong and controllable Rydberg-level interactions," Nat. Commun. 7, 13618 (2016).

12. D. Tiarks, S. Schmidt, G. Rempe, and S. Dürr, "Optical $\pi$ phase shift created with a single-photon pulse," Sci. Adv. 2, e1600036 (2016).

13. H. Busche, P. Huillery, S. W. Ball, T. Ilieva, M. P. A. Jones, and C. S. Adams, "Contactless nonlinear optics mediated by long-range Rydberg interactions," Nat. Phys. 13, 655 (2016).

14. Z. Bai and G. Huang, "Enhanced third-order and fifth-order Kerr nonlinearities in a cold atomic system via Rydberg-Rydberg interaction," Opt. Express 24, 4442 (2016).

15. H. Wu, Y. Li, Z. Yang, and S. Zheng, "Quantum signature for laser-driven correlated excitation of Rydberg atoms," Phys. Rev. A 95, 013842 (2017).

16. Q. Y. Liang, A. V. Venkatramani, S. H. Cantu, T. L. Nicholson, M. J. Gullans, A. V. Gorshkov, J. D. Thompson, C. Chin, M. D. Lukin, and V. Vuletić, "Observation of three-photon bound states in a quantum nonlinear medium," Science 359, 783 (2018).

17. Z. Bai, W. Li, and G. Huang, Stable single light bullets and vortices and their active control in cold Rydberg gases," Optica 6, 309 (2019).

18. S. Bai, X. Han, J. Bai, Y. Jiao, J. Zhao, S. Jia, and G. Raithel, "Cesium $n D_{J}+6 S_{1 / 2}$ Rydberg molecules and their permanent electric dipole moments," Phys. Rev. Res 2, 033525 (2020).
19. D. Ding, H. Busche, B. Shi, G. Guo, and C. S. Adams, "Phase diagram and self-organizing dynamics in a thermal ensemble of strongly interacting Rydberg atoms," Phys. Rev. X 10, 021023 (2020).

20. C. Fan, D. Rossini, H. Zhang, J. Wu, M. Artoni, and G. C. La Rocca, "Discrete time crystal in a finite chain of Rydberg atoms without disorder," Phys. Rev. A 101, 013417 (2020).

21. M. Zhou, J. Liu, P. Sun, Z. An, J. Li, X. Bao, and J. Pan, "Experimental creation of single Rydberg excitations via adiabatic passage,” Phys. Rev. A 102, 013706 (2020).

22. K. Liao, H. Tu, S. Yang, C. Chen, X. Liu, J. Liang, X. Zhang, H. Yan, and $\mathrm{S}$. Zhu, "Microwave electrometry via electromagnetically induced absorption in cold Rydberg atoms," Phys. Rev. A 101, 053432 (2020).

23. M. Jing, Y. Hu, J. Ma, H. Zhang, L. Zhang, L. Xiao, and S. Jia, "Quantum superhet based on microwave-dressed Rydberg atoms," Nat. Phys. 16, 911 (2020).

24. Y. Liu, Y. Sun, Z. Fu, P. Xu, X. Wang, X. He, J. Wang, and M. Zhan, "Infidelity induced by ground-Rydberg decoherence of the control qubit in a two-qubit Rydberg-blockade gate," Phys. Rev. Appl. 15, 054020 (2021).

25. J. Sinclair, D. Angulo, N. Lupu-Gladstein, K. Bonsma-Fisher, and A. M. Steinberg, "Observation of a large, resonant, cross-Kerr nonlinearity in a cold Rydberg gas," Phys. Rev. Res. 1, 033193 (2019).

26. C. Chen, F. Yang, X. Wu, C. Shen, M. K. Tey, and L. You, "Two-color optical nonlinearity in an ultracold Rydberg atom gas mixture," Phys. Rev. A 103, 053303 (2021).

27. M. Moreno-Cardoner, D. Goncalves, and D. E. Chang, "Quantum nonlinear optics based on two-Rydberg atom arrays," arXiv:2101.01936 (2021).

28. S. Baur, D. Tiarks, G. Rempe, and S. Dürr, "Single-photon switch based on Rydberg blockade," Phys. Rev. Lett. 112, 073901 (2014).

29. W. Li and I. Lesanovsky, "Coherence in a cold-atom photon switch," Phys. Rev. A 92, 043828 (2015).

30. H. Gorniaczyk, C. Tresp, P. Bienias, A. Paris-Mandoki, W. Li, I. Mirgorodskiy, H. P. Büchler, I. Lesanovsky, and S. Hofferberth, "Enhancement of Rydberg-mediated single-photon nonlinearities by electrically tuned Fórster resonances," Nat. Commun. 7, 12480 (2016).

31. C. R. Murray and T. Pohl, "Coherent photon manipulation in interacting atomic ensembles," Phys. Rev. X 7, 031007 (2017).

32. D. Tiarks, S. Baur, K. Schneider, S. Dürr, and G. Rempe, "Single-photon transistor using a Förster resonance," Phys. Rev. Lett. 113, 053602 (2014).

33. F. Ripka, H. Kübler, R. Löw, and T. Pfau, "A room-temperature single-photon source based on strongly interacting Rydberg atoms," Science 362, 446 (2018).

34. Y. Silberberg, "Collapse of optical pulses," Opt. Lett. 15, 1282 (1990).

35. Y. S. Kivshar and G. P. Agrawal, Optical Solitons: From Fibers to Photonic Crystals (Academic, 2006).

36. M. Belić, N. Petrović, W.-P. Zhong, R.-H. Xie, and G. Chen, "Analytical light bullet solutions to the generalized $(3+1)$-dimensional nonlinear Schrödinger equation," Phys. Rev. Lett. 101, 123904 (2008).

37. Y. V. Kartashov, B. A. Malomed, and L. Torner, "Solitons in nonlinear lattices," Rev. Mod. Phys. 83, 247 (2011).

38. Q. Zhang, Z. Bai, and G. Huang, "Fast-responding property of electromagnetically induced transparency in Rydberg atoms," Phys. Rev. A 97, 043821 (2018).

39. S. Mauger, J. Millen, and M. P. A. Jones, "Spectroscopy of strontium Rydberg states using electromagnetically induced transparency," J. Phys. B 40, F319 (2007).

40. The frequency and wave number of the probe field are given by $\omega_{p}+\omega$ and $k_{p}+K(\omega)$, respectively. Thus, $\omega=0$ corresponds to the center frequency of the probe field.

41. Z. Chen and G. Huang, "Trapping of weak signal pulses by soliton and trajectory control in a coherent atomic gas," Phys. Rev. A 89, 033817 (2014).

42. In the related numerical simulation, a $10 \%$ random disturbance has been added into the initial condition. 